\title{
Phase resonances on metal gratings of identical, equally spaced alternately tapered slits
}

\author{
Helen J. Rance, ${ }^{\text {a) }}$ Oliver K. Hamilton, J. Roy Sambles, and Alastair P. Hibbins \\ Electromagnetic Materials Group, School of Physics, University of Exeter, Exeter EX4 4QL, \\ United Kingdom
}

(Received 29 April 2009; accepted 13 June 2009; published online 29 July 2009)

\begin{abstract}
Phase resonances are observed in the microwave response of metal transmission gratings comprised of identical but alternately orientated tapered slits. Despite each slit having identical dimensions, and being equally spaced from its neighbors, these high-Q mode are nonetheless supported even at normal incidence. The excitation of these modes is attributed to the interference between FabryPerot-like modes and diffractively coupled surface waves. () 2009 American Institute of Physics. [DOI: $10.1063 / 1.3176408]$
\end{abstract}

The electromagnetic response of gratings with subwavelength-width holes ${ }^{1}$ and slits ${ }^{2}$ has attracted much theoretical and experimental interest in recent years due to the enhanced transmission phenomena they exhibit. Most authors have concluded that these effects can be attributed to the excitation of bound surface waves (e.g., surface plasmons $^{3}$ at the metal/dielectric interface) as well as coupling to localized cavity modes. ${ }^{2}$ Several workers have analyzed the relative roles of different types of surface modes and the cavity resonances they induce in producing enhanced transmission and absorption. ${ }^{2,45}$ For example, consider a metal slit illuminated with electromagnetic radiation whose electric vector lies perpendicular to the groove direction. In this geometry, the walls defining the cavity will support a surface wave. ${ }^{6}$ At microwave frequencies metals behave as near-perfect conductors, almost totally excluding the incident field. However as the slit width is reduced, the fields of the surface waves on opposite walls couple together across its width. In the limit of infinite conductivity ${ }^{7}$ this coupled surface mode is entirely equivalent to the transverse electromagnetic waveguide mode supported by each slit. ${ }^{8}$ For finite length slits, the mode is quantized to yield a family of Fabry-Perot (FP)-like cavity modes, the resonant frequencies of which yield transmission peaks which satisfy the condition $\nu_{N} \approx c N / 2 t$ where $t$ is the thickness of the metal substrate. The response of such gratings formed from unit cells comprising of a single slit exhibit one resonance per harmonic $(N)$. However periodic arrays of resonators in which the primitive unit cell comprises of more than one element (compound gratings) are known to support additional resonances, since the associated structure factor introduces new degrees of freedom regarding the possible eigenmodes. ${ }^{9-14}$ These so called "phase resonances" are simply a coupled resonator phenomenon, observed as a redistribution of the available energy between propagating orders.

The structure factor in the grating under investigation in this study, (Fig. 1, inset) is created from alternating the orientation of the slits. Significantly the slits are identical and equally spaced. Previous workers however have considered compound gratings in which identical cavities are unequally spaced under normal ${ }^{12}$ and oblique illumination, ${ }^{13}$ as well as gratings with no structural symmetry in their basic units. ${ }^{14}$

${ }^{a)}$ Electronic mail: h.j.rance@ex.ac.uk.
By recording data as a function of the angle of incidence, insight has been obtained about the band structure of the resonances supported by our structure. This highlights the importance of interference between the surface modes that exist on the top and bottom surfaces of the array (diffractively coupled surface waves), and the FP-like modes, when considering the origin of the high-Q modes supported by the grating in this study, even at normal incidence illumination.

The experimental sample (Fig. 1 inset) is constructed by stacking together approximately $100500 \mathrm{~mm}$ long aluminum-alloy metal slats, of thickness $t=19.8 \mathrm{~mm}$ and width $w=3.00 \mathrm{~mm}$, separated from one another by a tapered-width air-filled slit using small polyester spacers outside of the area illuminated by the incident beam. The unit cell of the structure comprises of two metal lengths, spaced from one another by $a=1.5 \mathrm{~mm}$ at one end, and $b=0.5 \mathrm{~mm}$ at the opposite end. The grating therefore has a periodicity of $\lambda_{g}=7.99 \mathrm{~mm}$, with a plane of mirror symmetry in the center of each air-filled slit. Microwave radiation is incident upon the experimental sample in the $x z$-plane at an angle $\theta$ to the surface normal, and is $p$-polarized. The sample is placed on a computer controlled rotating table midway between the two spherical mirrors. A microwave source sweeps frequency $5 \leq \nu \leq 40 \mathrm{GHz}$ and feeds a fixed position horn antenna. This source antenna is placed at the focus of the first mirror, and the reflected collimated beam is directed onto the grating sample. The transmitted nondiffracted beam

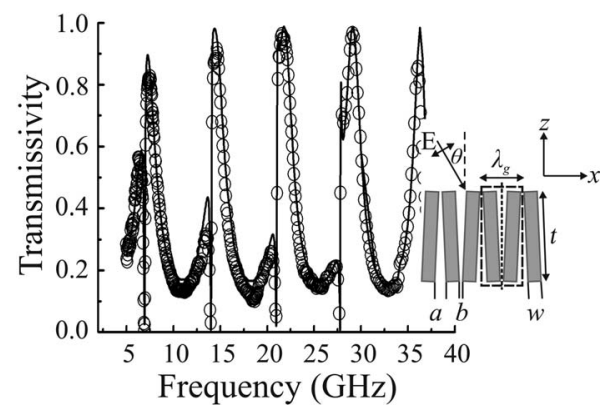

FIG. 1. Experimental transmissivity (circles) for normal incidence $(\theta=0)$. The solid line represents predictions from the numerical model. Inset: Sample geometry and coordinate system of the experimental structure. The slats have length of approximately $500 \mathrm{~mm}$ in the $y$-direction. 


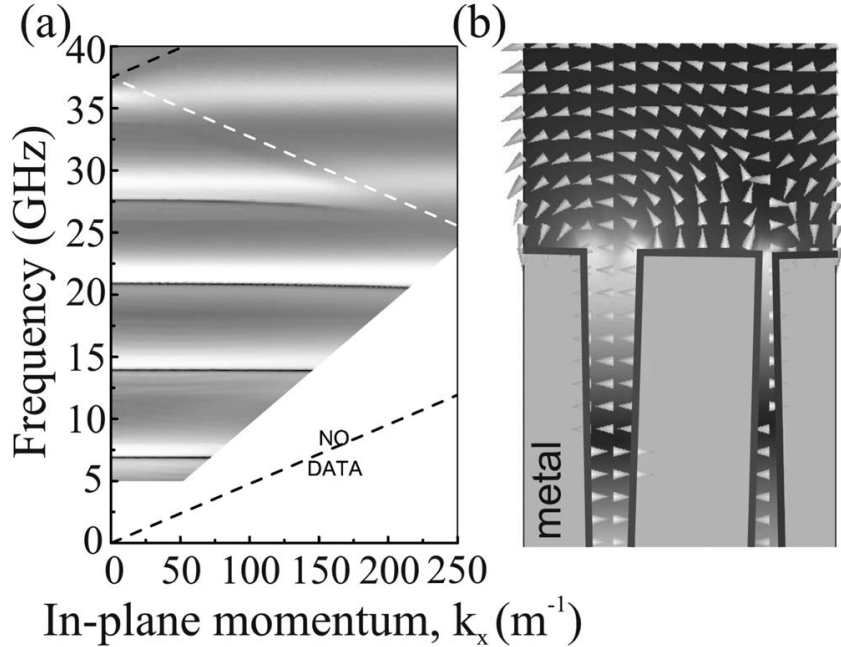

FIG. 2. (a) Experimental transmissivity plotted as a function of $k_{x}$ $=(2 \pi \nu / c) \sin \theta$. White regions correspond to normalized transmissivites in excess of $90 \%$. The dotted black and white lines correspond to the light line and diffracted light line. (b) Predictions of the electric field distribution in the $x z$-plane on resonance with normal incidence illumination $(\theta=0)$. The gray scale illustrates time-averaged electric-field magnitude and the arrow heads indicate the direction of the electric field vector at a phase in its temporal cycle corresponding to a maximal intensification. The +- mode occurs at $\nu=14.05 \mathrm{GHz}$. The lightest shading corresponds to a field enhancement of at least 15 times.

is collected by the second mirror and is focused to a matched detector horn.

Figure 1 illustrates the normalized experimental transmissivity at normal incidence together with predictions from the numerical model. ${ }^{15}$ The sample is rotated out of the $x z$-plane by $\sim 3^{\circ}$ to reduce the fast oscillations associated with back reflections between the sample and mirror. The transmission spectrum is characterized by a series of broad resonant peaks each perturbed by a phase resonance on its low frequency side. Finite element method ${ }^{15}$ predictions of the electric fields on resonance have been examined at the two resonant conditions associated with each harmonic $(N)$. The first we label " ++ ," where the resonance of each slit is driven in phase with the incident field (not shown). This resonance is equivalent to the FP-like mode of a simple transmission grating of narrow slits. The second (lower frequency) Fano-like resonance is associated with a "+ -" nearfield phase configuration, in which the phase of the resonant fields in neighboring slits differ by $\pi$ radians (Fig. 2). It is important to note that similar phenomena have only previously been observed in two-cavity compound gratings, in which the cavities differ in width, ${ }^{14}$ or structures of identical but unequally spaced cavities with nonzero angles of illumination. ${ }^{13}$

The origin of the phase resonance can be explored by considering the photonic band structure of diffractively coupled surface waves supported on the faces of the metallic grating. Of course, while surface waves propagating on planar substrates in the microwave regime are essentially grazing photons (owing to the near-infinite conductivities of metals), in the present study these modes very significantly perturbed. However, we can understand much about the response of our structure by considering only the two dominant scattering terms associated with the grating profile (Fig. 3). These are, a large amplitude component associated with the spacing of the slits $\left(\lambda_{g}=2 \pi / k_{S}\right)$, convolved with a weaker

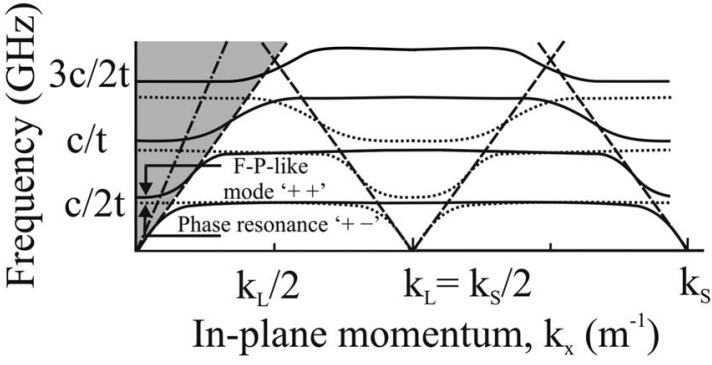

FIG. 3. Schematic representation of the dispersion of the modes within the first three harmonics supported by our experimental sample. The dashed lines correspond to the light line and diffracted lights lines. The shaded area bordered by the light line represents the region of momentum space within which an incident photon may couple to a mode and the area left of the dot-dashed line corresponds to the region in which experimental data has been taken. The solid lines illustrate the dispersion of the FP-like mode $(++)$ scattered by the grating wave vector $k_{S}$ and the dotted lines illustrate the dispersion of the phase resonance mode $(+-)$ scattered by the grating wave vector $k_{L}$.

component associated with the longer pitch of the unit cells $\left(\Lambda_{g}=2 \lambda_{g}=2 \pi / k_{L}\right)$. The strong, short-pitch component $\left(k_{S}\right)$ of the grating profile creates large gaps in the mode's dispersion (which would otherwise follow the light line) at the corresponding Brillouin zone boundaries $\left(k_{x}=0\right.$ and $\left.k_{S} / 2=k_{L}\right)$. A schematic illustration of the resulting band structure is shown in Fig. 3 (solid line). (A useful and detailed discussion of the band structure of surface modes on deep gratings can be found in Ref. 16). While the lowest energy mode exists outside the incident light line, the higher order diffractively coupled modes are found inside the radiative region. They cross the $k_{x}=0$ axis at the FP frequencies $\left(\nu_{N}=C N / 2 t\right)$, at which the mode becomes strongly localized within the slit. These are the origin of the broad transmission peaks in Fig. 1 , i.e., the mode associated with the ++ phase configuration. Note also that the dispersion that results may equivalently be considered as a series of anticrossings between the eigenmodes of the slit cavities and the photonlike surface wave.

By rotating the sample to vary the in-plane momentum $k_{x}=(2 \pi \nu / c) \sin \theta$, we map the flat photonic band nature of the modes supported by our sample [Fig. 2(a)]. It is clear that two modes are observed in the photonic band structure per harmonic; the first being the aforementioned FP-like mode. The second mode, which we will subsequently show is associated with the +- phase configuration [Fig. 2(b)], always occurs at a lower frequency. The existence and dispersion of these much narrower modes can be understood from a further band-folding discussion. While the magnitude of the scattering associated with $k_{L}$ is too weak to cause much perturbation of the surface wave dispersion curve, sections of the dispersion curve that would exist outside of the incident light line for a grating of pitch $\lambda_{g}$ are folded into the radiative region (see Fig. 3). Hence the originally nonradiative modes associated with the low energy edge of the band gap created by the short-pitch component become excitable through scattering introduced by the long-pitch component. The resulting standing wave solution on the surface of the grating therefore has the expected wavelength equal to $\Lambda_{g}$. These resonances are narrower than the strongly scattered primary mode. Furthermore, it is clear that they must always occur at a lower frequency than the primary mode since the upper band edge of the $N$ th mode can never overlap with the lower band edge of the $(N+1)$ th mode. ${ }^{17}$ From closer examination of Fig. 3 , it 


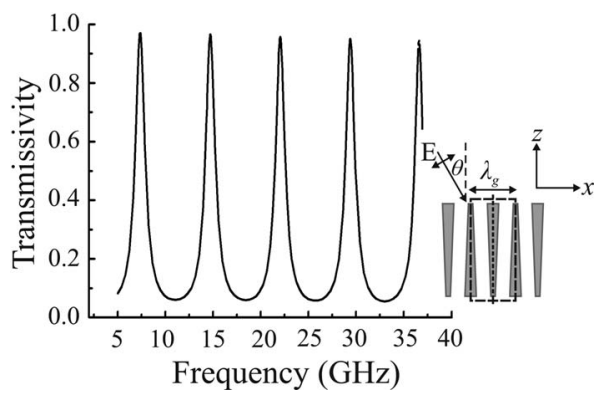

FIG. 4. Model transmissivity for the "inverse" structure illuminated at normal incidence $(\theta=0)$.

is also evident that the frequency separation between the ++ and +- modes increases with order $N$. This is associated with the reducing separation (in $k_{x}$ ) between the light line (with which the surface modes strongly interact) and the requirement for the modes to have zero gradient (group velocity) at the Brillouin zone boundary.

The Fano-like resonances associated with the +- phase condition are due to the interference between two competing transmission channels. The first transmission mechanism is direct coupling into the radiative FP-like modes of the slits, producing the broad, flat-banded peaks (as observed from simple, deep gratings). The second transmission mechanism involves first coupling to a surface wave on the front face of the array, which in turn interacts through the FP-like resonance with an identical surface wave on the opposite face of the array, which subsequently reradiates. Coupling into and out of the first and second surface waves, respectively, induces a phase shift of $\sim \pi / 2$ radians each time, ${ }^{16}$ resulting in the Fano-shaped resonance observed.

It is interesting to note that numerical modeling of the "inverse" structure in which the metal slats are tapered reveals that it does not support a phase resonance for normal incidence; the transmission response is characterized by a series of simple, broad FP-like resonant peaks (Fig. 4). This difference arises from the fact that adjacent cavity entrances are not of equal size. Hence for the mode in which the fields at those entrances points are in opposite directions, there exists a possibility of coupling at normal incidence. This is clearly not possible for the inverse structure, when the adjacent cavities have identical entrance sizes. Thus the inverse structure is similar to those studied in Ref. 13 for which in order to support a phase resonance, the symmetry of the system has to be broken by a nonzero angle of illumination.
Conversely, our original experimental sample, which has a mirror plane of symmetry in the dielectric, is similar to the structures in Ref. 14 that rely on the inherent geometry of the structure breaking the symmetry of the system, allowing phase resonances to be supported even with normal incidence illumination.

To summarize, we have studied the electromagnetic response of compound gratings in which each cavity is identical (therefore has the same resonant frequency) and is equally spaced from its neighbor. We create a structure factor by changing the orientation of alternate tapered slits, unlike in previous studies which rely on variation of the slit widths ${ }^{11,14}$ or the spacing between them. ${ }^{9,12}$ Thus, the phase resonance supported by our experimental sample, even at normal incidence, can only be attributed to interference between simple FP-like transmission, and an additional transmission channel that we associate with the excitation of surface waves propagating across the face of the array of slits.

The authors would like to acknowledge the financial support of the Engineering and Physical Sciences Research Council (EPSRC, U.K.).

${ }^{1}$ T. W. Ebbesen, H. J. Lezec, H. F. Ghaemi, T. Thio, and P. A. Wolff, Nature (London) 391, 667 (1998).

${ }^{2}$ J. A. Porto, F. J. García-Vidal, and J. B. Pendry, Phys. Rev. Lett. 83, 2845 (1999).

${ }^{3}$ W. L. Barnes, A. Dereux, and T. W. Ebbesen, Nature (London) 424, 824 (2003).

${ }^{4}$ S. Collin, F. Pardo, R. Teissier, and J. Pelouard, J. Opt. A: Pure Appl. Opt. 4, S154 (2002).

${ }^{5}$ D. C. Crouse, E. Jaquay, and A. Maikal, Phys. Rev. B 77, 195437 (2008).

${ }^{6}$ H. E. Went, A. P. Hibbins, J. R. Sambles, C. R. Lawrence, and A. P. Crick, Appl. Phys. Lett. 77, 2789 (2000).

${ }^{7}$ Y. Takakura, Phys. Rev. Lett. 86, 5601 (2001)

${ }^{8}$ A. P. Hibbins, M. J. Lockyear, and J. R. Sambles, J. Appl. Phys. 99, 124903 (2006).

${ }^{9}$ A. N. Fantino, S. I. Grosz, and D. C. Skigin, Phys. Rev. E 64, 016605 (2001).

${ }^{10}$ D. C. Skigin and V. V. Veremey, IEEE Trans. Antennas Propag. 47, 376 (1999).

${ }^{11}$ A. P. Hibbins, I. R. Hooper, M. J. Lockyear, and J. R. Sambles, Phys. Rev. Lett. 96, 257402 (2006).

${ }^{12}$ D. C. Skigin and R. A. Depine, Phys. Rev. Lett. 95, 217402 (2005).

${ }^{13}$ D. C. Skigin and R. A. Depine, Phys. Rev. E 74, 046606 (2006).

${ }^{14}$ Y. G. Ma, X. S. Rao, G. F. Zhang, and C. K. Ong, Phys. Rev. B 76, 085413 (2007).

${ }^{15}$ www.ansoft.com.

${ }^{16}$ I. R. Hooper and J. R. Sambles, Phys. Rev. B 65, 165432 (2002).

${ }^{17}$ W.-C. Tan, T. W. Preist, J. R. Sambles, and N. P. Wanstall, Phys. Rev. B 59, 12661 (1999). 